\title{
Interstitial ectopic pregnancy following ipsilateral salpingectomy
}

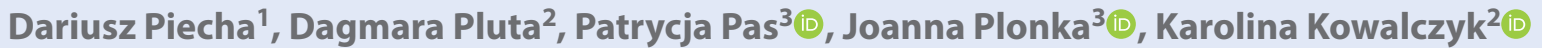 \\ ${ }^{1}$ Department of Obstetrics and Gynaecology, Medical Center in Piekary Slaskie, Poland \\ ${ }^{2}$ Clinic of Gynecological Endocrinology, Department of Gynecology and Obstetrics, \\ Medical University of Silesia, Katowice, Poland \\ ${ }^{3}$ Gynecological Endocrinology Students Scientific Association, Medical University of Silesia, Katowice, Poland
}

A 32-year-old woman (gravida 3, para 1) was referred to the Department of Gynecology with suspected ectopic pregnancy six weeks after her last menstrual period. Her medical history revealed one cesarean section with concomitant intramural fibroid resection 18 months earlier. Then, after 10 months, she experienced an ectopic pregnancy in the right fallopian tube. Because of this, a laparoscopy was performed and the whole tube with gestational sac was removed due to isthmus and interstitial part adhesions.

On admission the patient was asymptomatic, having neither abdominal pain nor uterine bleeding. A physical exam revealed benign abdomen, normal uterine size and non-palpable adnexa. Gradual increase in beta human chorionic gonadotropin ( $\beta$-hCG) was observed $-415 \mathrm{mlU} / \mathrm{mL}$ in the fourth week of pregnancy and $5000 \mathrm{mlU} / \mathrm{mL}$ in the fifth week. After admission, two-day increase from $9384 \mathrm{mlU} / \mathrm{mL}$ to $16345 \mathrm{mlU} / \mathrm{mL}$ was observed. However, there was no gestational sac inside the uterine cavity visible on the transvaginal ultrasound. A $30 \times 15 \mathrm{~mm}$ heterogeneous mass with hypoechoic central part and a peripheral vascular rim in Power Doppler ultrasound was observed in the right cornual area.

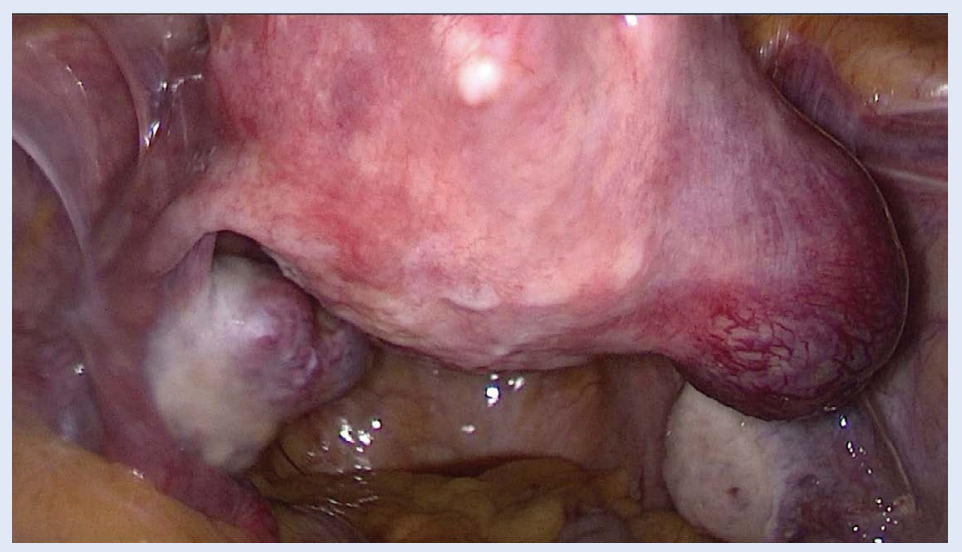

Figure 1. Right interstitial ectopic pregnancy with visible myometrial trophoblast invasion
The patient was scheduled for exploratory laparoscopy which showed an interstitial ectopic pregnancy in the right uterine horn with a diameter of $2-3 \mathrm{~cm}$ (Fig. 1). Due to massive pelvic adhesions a decision was made to proceed with a conversion to laparotomy. A successful right-sided cornual resection with removal of interstitial ectopic pregnancy tissue was performed. Uterine closure was performed with double-layer suturing. Anti-adhesion prophylaxis was applied. The patient made an uneventful postoperative recovery and was discharged from the hospital 3 days after the surgery.

Interstitial (cornual) pregnancy refers to an ectopic pregnancy that is implanted in the tubal segment traversing the muscular wall of the uterus. This section of tube is relatively thick, and is located in a highly vascular region. Therefore, interstitial ectopic pregnancy tends to rupture later, with more severe bleeding than other ectopic pregnancies. Its incidence accounts for $2-4 \%$ of ectopic pregnancies. Additionally, the mortality rate varies from 2.0 to $2.5 \%$. The high mortality in this type of pregnancy is partially because of delay in diagnosis and the speed of hemorrhage. The etiology of interstitial pregnancy remains unknown. However, similar risk factors for tubal ectopic pregnancy such as pelvic inflammatory disease, tubal disease, adnexal surgery, and assisted reproductive techniques are associated [1]. The risk of reappearance of ectopic pregnancy is approximately $15 \%$ and increases to $30 \%$ with two previous ectopic pregnancies [2]. Recurrent ipsilateral spontaneous ectopic pregnancy after total salpingectomy is a very rare occurrence.

There are several described mechanisms of recurrent ipsilateral ectopic pregnancy. The first one involves the spermatozoa or embryo passing through the contralateral patent tube into the pouch of Douglas and then migrating to the damaged fallopian tube. A second theory suggests the passage of the fertilized egg through the contralateral intact uterine tube. It is also possible that despite surgical excision of the tube after salpingectomy; the lumina remain intact in the interstitial portion and

Corresponding author:

Karolina Kowalczyk

Clinic of Gynecological Endocrinology, Department of Gynecology and Obstetrics, Medical University of Silesia, Katowice, Poland

e-mail:karolina.kowalczyk74@gmail.com 
distal remnant of the fallopian tube, allowing communication between the endometrial and peritoneal cavities. This allows for fertilization and implantation within this portion of the remnant tube [3].

The literature reports a high variety of interstitial pregnancy treatment regimens. Surgical management is indicated in the presence of significant symptoms, cornual rupture or large pregnancies. A trend in the last few years has been to use conservative treatment such as laparoscopic cornual resection or cornuostomy if possible [4]. In our patient, recurrent ectopic pregnancy and massive pelvic adhesions justified the protocol of cornual resection via laparotomy. Methotrexate injection is used as a successful noninvasive option - it is administered either systemically or locally [5].

A cornual gestation is one of the most hazardous types of ectopic gestation. The diagnosis and treatment of such a pregnancy is challenging and constitutes an urgent medical situation. Early clinical diagnosis of ectopic pregnancy based on the combination of a clinical suspicion, serum $\beta$-hCG assays, transvaginal ultrasonography findings supported by laparoscopy may help to contribute towards effective conservative treatment options.

\section{REFERENCES}

1. Pramayadi CT, Bramantyo A, Gunardi ER. Successful Procedure in Conservative Management of Interstitial (Cornual) Ectopic Pregnancy. Gynecol Minim Invasive Ther. 2018; 7(4): 172-174, doi: 10.4103/GMIT.GMIT_9 18, indexed in Pubmed: 30306037.

2. Diagnosis and Management of Ectopic Pregnancy. RCOG Green Top Guidelines . 2016; 21.

3. Gao MYi, Zhu H, Zheng FY. Interstitial Pregnancy after Ipsilateral Salpingectomy: Analysis of 46 Cases and a Literature Review. J Minim Invasive Gynecol. 2020; 27(3): 613-617, doi: 10.1016/j.jmig.2019.04.029, indexed in Pubmed: 31589932.

4. Cucinella G, Calagna G, Rotolo S, et al. Interstitial pregnancy: a,road map' of surgical treatment based on a systematic review of the literature. Gynecol Obstet Invest. 2014; 78(3): 141-149, doi: 10.1159/000364869, indexed in Pubmed: 25060047.

5. Nikodijevic K, Bricou A, Benbara A, et al. Cornual pregnancy: Management and subsequent fertility. Gynecol Obstet Fertil. 2016; 44(1): 11-16, doi: 10.1016/j.gyobfe.2015.10.011, indexed in Pubmed: 26678164. 